A NOTE ON THE

\section{CLINICAL DIAGNOSIS OF DIPHTHERIA AND OTHER EXUDATIONS IN THE THROAT. ${ }^{1}$}

BY HARRY DRINKWATER, M.D. EDIN., PHYSICIAN TO THE WREXHAM FEVER HOSPITAL.

BY clinical diagnosis I mean immediate cliagnosis, at the bedside of the patient, by ocular inspection of the fauces, before a bacteriological examination is made.

A very considerable percentage of cases certified as diphtheria and sent as such into the fever hospital of which I have been in charge for between three and four years have been cases of follicular tonsillitis, influenza, or simple catarrh. On the other hanc, many cases have been admitted in a hopeless condition of toxæmia, or only just in time for life to be saved by tracheotomy, the disease having been treated for several days as "quinsy." This uncertainty as to the differentiation of diphtheria from the simpler infections of the fauces is widespread, and medical practitioners are often in doubt apart from a bacteriological examination, which may mean a delay of 24 or 48 hours, with possibly disastrous results.

The thesis which $I$ advance is that if certain visible characteristics are carefully observed, and more especially the distribution of the disease patches in the fauces, this uncertainty should seldom occur. As a matter of fact, my naked-eye diagnosis has been confirmed in every case by subsequent bacteriological examination, with the single exception of Vincent's angina, which cannot always be distinguished from diphtheria by the eye alone. In from 10 to 20 per cent. of cases of true diphtheria an examination of a swabfilm is negative, and the Klebs-Löffler bacillus is only detected after cultivation in an incubator. Very often the swab has to be sent away by post, and there is inevitable delay. It is, therefore, obvious that if a diagnosis can be made by ocular inspection alone such means of diagnosis must have an immense advantage over the bacteriological method, however desirable the latter plan may be for purposes of confirmation.

If my plan of diagnosis has been uniformly correct for a period of over three years, in a hospital serving a district with a population of about 80,000 , one is driven to the conclusion that exceptions must be few and far between. The points to be relied upon in making the diagnosis are illustrated by the drawings here shown; they are all hospital cases admitted as diphtheria.

The first point to note is the distribution of the patches, and for this purpose the fances may be considered as divisible into six areas, three on each side of the mid-line, as shown in Fig. 1, where $A B$ is the mid-line and the figures $1,2,3$ are placed in the tonsillar, the uvular, and the palatal areas respectively. Every area may show some deposit, and in any one area the deposit may consist of a single patch or of several patches. The tonsillar area is the one most frequently affected. Now the important thing to observe is whether the tonsillar area (or any other area) shows one patch or more than one. If two or more patches are present on one tonsil or in one tonsillar area the disease is neither diphtheria nor Vincent's angina; most frequently it is follicular tonsillitis or influenza. In diphtheria the patch in any one area is single, and the same is true of Vincent's angina; in follicular tonsillitis and in influenza there are multiple patches in the tonsillar area.

In diphtheria the patch shows certain well-marked characteristics-viz.: (1) It is raised above the level of the mucous membrane. (2) The edges of the deposit are sharply defined all round. (3) The colour varies greatly; it may be white and glistening, bluish, yellow, or spotted with black or red. It rarely has the "wet wash-leather" appearance described in the textbooks.

In a given case of membranous deposit on the fauces, if it is raised above the level of the mucous membrane, has sharply defined edges, and is single in each affected

1 Rased upon a paper introducing a discussion at the Medical society of London on Feb. 9th (see THE LANCWT, Feb. 21st, p. 442) area, there can be no doubt that the disease is either diphtheria or Vincent's angina, and this diagnosis is arrived at in a few seconds without delay in waiting for a bacteriological report.

It is necessary to remark that these features are characteristic of the disease in the period of growth; after resolution has begun and the inembrane is being cast off a number of isolated islands may be seen in the tonsillar area; but, of course, the diagnosis should have been made before this stage is reached. In diphtheria or Vincent's angina there may be five or six patches, but in neither disease do we see (in the period of growth) more than one patch in any one of the six faucial areas; each area may have one patch, but not more than one. If, then, in a given case two or more patches are seen in the tonsillar area the disease is not diphtheria, and a bacteriological examination is not required except for confirmation of the naked-eye diagnosis. The term diphtheria is applied to those cases in which Klebs-Löffler or Hoffmann's bacilli are present. If these germs are not present the disease is regarded as other than diphtheria.

\section{Descriptions of Illustrations.}

FIG. 1.-The areas of the fauces: (1) tonsillar area, between anterior and posterior pillars of the fances; (2) uvular area

(3) palatal area.
Fr(t. 2,--Diphtheria. Single sharply defined vatch in each tonsillar FIr. 2,- Diphtheria. Sine
area ; tonsils swollen.

area ; tonsils swollen.
FIt. 3.-Diphtheria. One patch in each tonsillar area; shows elevation of patch above surface of mucous membrane.

Figr. 4.-Diphtheria. Single pateh on each side.

FIg. 5.-Diphtheria. Thick membrane extending into all six areas edges sharply defined.

Fig. 6.-Diphtheria. Involving every area except right palatal. FIG. 7.-Hoffmann's diphtheria. Distinguishable from Klebs Löffler diphtheria only by bacteriological examination.

Fra. 8.-Vincent's angina The distribution is like that of diphtheria. The deep depression ("penetrating ulcer") in the patch on the left tonsil is characteristic of Vincent's angina.

FIG. 9.-Vincent's angina The thinning of the lower part of each patch is characteristic of Vincent's ansina. It is not seen in diphtheria.

FIC. 10.-Double infection of diphtheria and Vincent's angina. FT. 11.-Double infection of diphtheria and Vincent's angina. FIG. 12.--Influenza. Distinguished from diphtheria and Vincent's angina by having more than one patch in the tonsillar area. FiG. 13. - Influenza. Showing two patches on right tonsil.

Fra. 14.-Influenza. The two patches on the left tonsil at once distinguish it from diphtheria.

Fig. 15.-Follicular tonsillitis. Tonsils consested. Pus oozing out IG. 15.-Follicular tonsillitis. Tonsils consested. Pus oozing ou
of several follicles. FIa. 16. - Scarlet fever. FIG. 17.-Rubella. of several follicles. FIG. 16.-Scarlet fever, FIG. 17.-Rnbella
Fig. 18.-Secondary syphilis. Thin deposit. The case was one of If. 18.-Secondary syphilis. Thin deposit. The case was one of
diphtheria which showed this appearance three weeks after adphtheria which sho

The appearance of Vincent's angina as seen in the throat is very similar to that of diphtheria, so similar, in fact, that in many cases it is quite impossible to differentiate the two diseases. Vincent's angina may be said to mimic diphtheria, just as syphilis and hysteria mimic other diseases. Occasionally, however, we come across a case of Vincent's angina which can be at once recognised as such, owing to certain special features, the most characteristic of which is a sharply defined penetrating ulcer about the middle of the patch with its long axis running vertically, the deposit some times scarcely extending beyond the margins of the ulcer. Another peculiar feature is the marked thinning of the lower portion of the patch, making the inferior limit ill-defined. If either of these features be present Vincent's angina may be safely diagnosed. There is sometimes considerable erosion of the uvula. Sometimes the deposit is softer and more creamy than in diphtheria, whilst in other instances it is extremely dense and tenacious.

In follicular tonsillitis there is marked swelling and congestion of the tonsils, and muco-pus can be seen oozing out of several follicles. The single patch characteristic of diphtheria and Vincent's angina is never seen. In influenza the patches are multiple in the tonsillar area, and very often without any appreciable swelling of the tonsils.

Should these points be generally confirmed the con clusion would follow that, from the point of view of practical medicine, the naked-eye diagnosis at the bedside of the patient is incalculably more important than the bacteriological diagnosis, and that if generally acted upon there will result a considerable saving of life. 
The Langer, ] DR. H. DRINKWATER : CLINICAL DIAGNOSIS OF DIPHTHERIA. [MAY 29, 19201161

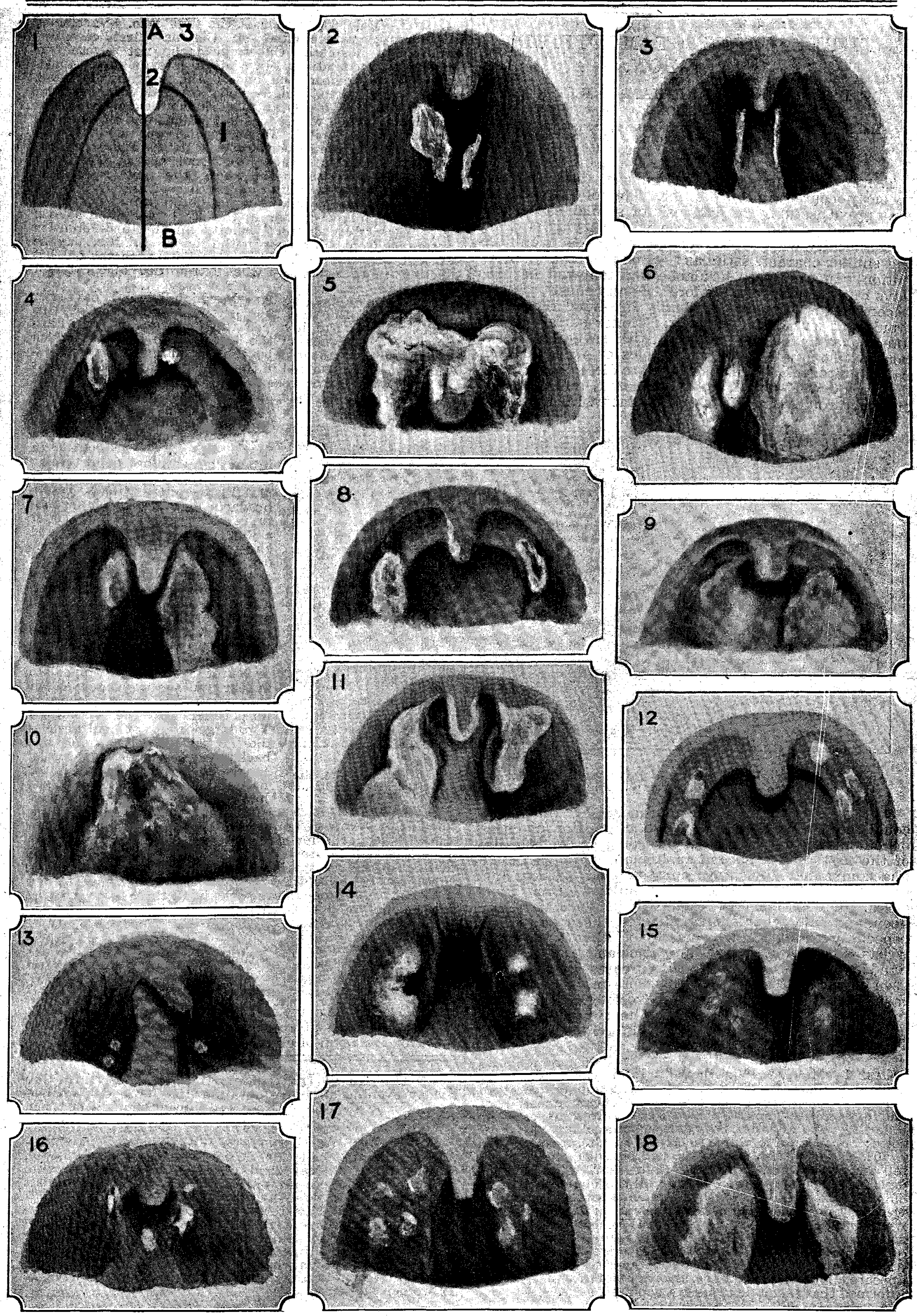

